Pacific Journal of Mathematics

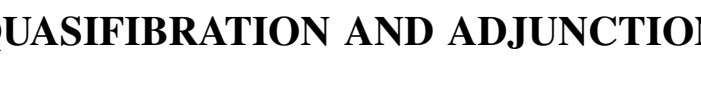




\title{
QUASIFIBRATION AND ADJUNCTION
}

\author{
K. A. HARDIE
}

This paper is concerned with the preservation of quasifibration under pair adjunction of Hurewicz fibrations, and the preservation of weak homotopy equivalence under pair adjunction of identity maps.

The foundations of the theory of quasifibrations were laid by Dold and Thom in their paper [3] in which they proved that the homotopy groups of the infinite symmetric product of a space $X$ were naturally isomorphic with the integral homology groups of $X$. Another application was soon given by Dold and Lashof [2] generalizing Milnor's construction [9] of a universal principal fibre bundle with given structure group and their results were further generalized by Stasheff [13], Milgram [8], Steenrod [15] and Stasheff [14]. Other applications of quasifibrations occur in [6], [5] and [1]. Since, as a generalisation of fibration, quasifibration has a serious deficiency (it is not preserved under pull-back) one may well ask why it has proved to be so useful. A study of the papers referred to reveals that it is essentially the behaviour of quasifibration with respect to adjunction which is involved. However, the relevant arguments mostly rely on a basic lemma of [3] (lemma 2.10) and proceed ad hoc.

Let $p: P \rightarrow P^{\prime}, t: T \rightarrow T^{\prime}, q: Q \rightarrow Q^{\prime}$ be pairs (i.e., continuous maps) and let $\phi=\left(f, f^{\prime}\right): p \rightarrow t, \gamma=\left(g, g^{\prime}\right): p \rightarrow q$ be pair maps and consider the push-out diagram

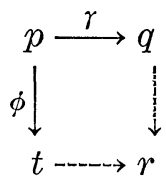

in the category of pairs. $\gamma$ is a weak homotopy equivalence of fibres (WHEF) if, for each $x \in P^{\prime}$, the induced map

$$
g^{\prime \prime}: p^{-1}(x) \longrightarrow q^{-1}\left(g^{\prime} x\right)
$$

is a weak homotopy equivalence. We shall prove the following theorem.

TheOREM 0.2. If $f^{\prime}$ is a closed cofibration, if $t$ is a fibration, if $p$ is the pull-back of $t$ over $f^{\prime}$, if $q$ is a quasifibration and if $\gamma$ is a WHEF then $r$ is a quasifibration. 
In most of the constructions of quasifibrations in the papers cited the situation is or is equivalent to the special case of 0.2 in which $t$ is a trivial fibration.

That 0.2 does not remain valid if "quasifibration" is replaced by "Hurewicz fibration" can be seen from the example suggested by the diagram.
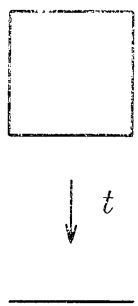


FIGURE 1
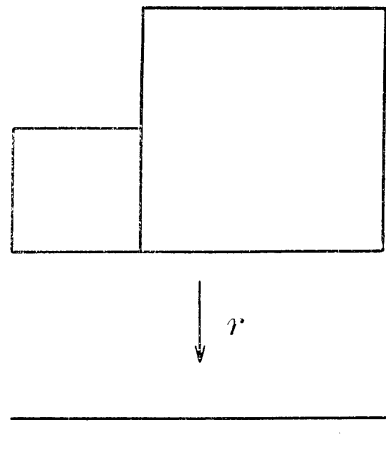

Let $1_{P}: P \rightarrow P$ denote the identity pair. Replacing $p$ by $1_{P}$ and $t$ by $1_{T}$ we shall obtain the following analogue of 0.2 for weak homotopy equivalences.

THEOREM 0.3. If $f$ is a closed cofibration, if $\phi=(f, f): 1_{P} \rightarrow 1_{T}$ and if $q$ is a weak homotopy equivalence then $r$ is a weak homotopy equivalence.

0.3 is particularly useful when the range and domain of $r$ are not 1-connected for then a proof via relative homology isomorphisms and the J.H.C. Whitehead theorem breaks down.

1. Mapping cylinders. We recall that the space $R=R(f, g)$ obtained by completing the push-out diagram

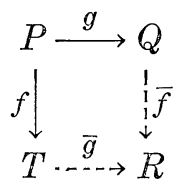

is that obtained from the topological sum $T \vee Q$ (disjoint union) of $T$ and $Q$ by factoring out by the relation

$$
f x \sim g x \quad(x \in P) .
$$

We shall denote the equivalence class of $y \in T \vee Q$ by $\{y\}$. Let ${ }^{-} i_{0}, i_{1}$ : $P \rightarrow P \times I$ be the maps given by 


$$
i_{0} x=(x, 0), i_{1} x=(x, 1) \quad(x \in P) .
$$

We recall that the mapping cylinder of $f$ is the space $Z_{f}=R\left(f, i_{1}\right)$. The projection of $P \times I$ on to $P$ induces a retraction $b: Z_{f} \rightarrow T$ and if we set $i=\bar{f} \cdot i_{0}$, we have $b \cdot i=f$ and $b \cdot \bar{i}_{1}=1_{T}$. The space $Z(f, g)=$ $R(i, g)$ has been called [10] the mapping cylinder of the cotriad $T \stackrel{f}{\longleftarrow} P \stackrel{g}{\longrightarrow} Q \quad(T f P g Q)$. The cotriad map $\left(b, 1_{P}, 1_{Q}\right):\left(Z_{f} i P g Q\right) \rightarrow$ (TfPgQ)



induces a map $k(f, g): Z(f, g) \rightarrow R$ and we have:

LEMMA 1.2. If $f$ (or $g$ ) is a cofibration then $k(f, g)$ is a homotopy equivalence.

Proof. If $f$ is a cofibration then $\left(1_{P}, b\right): i \rightarrow f$ is a homotopy equivalence of pairs. A proof for the reduced case but equally valid in our situation is given in [4; pp. 17, 18]. Then 1.2 is a consequence of the following lemma, the proof of which being straightforward is omitted. Let $(t, p, q):(T f P g Q) \rightarrow\left(T^{\prime} f^{\prime} P^{\prime} g^{\prime} Q^{\prime}\right)$ be a cotriad map. Then there is an induced map

$$
r: R(f, g) \longrightarrow R\left(f^{\prime}, g^{\prime}\right)
$$

and we remark that $r$ completes diagram 0.1. We have

LEMMA 1.3. If $(t, p, q)$ is a cotriad homotopy equivalence then $r$ is a homotopy equivalence.

REMARK 1.4. In view of [11; Satz 3], the conclusion of 1.2 remains valid if $f$ has instead the weak homotopy extension property (WHEP).

The cotriad map $(t, p, q)$ also induces a map $r_{z}: Z(f, g) \rightarrow Z\left(f^{\prime}, g^{\prime}\right)$ and we have:

LEMMA 1.5. If $t, p, q$ are homotopy equivalences then $r_{z}$ is a homotopy equivalence. If, further, $f$ and $f^{\prime}$ (or $f$ and $g^{\prime}$ ) have the WHEP then $r$ is a homotopy equivalence.

The proof of the first assertion of 1.5 will also be omitted since 
it is equivalent to a special case of $[10 ; 4.6]$, the proof of the dual $[10 ; 2.6]$ having been given in full. The first assertion combined with 1.2 yields the second assertion.

Similarly the pair map $(p, t): f \rightarrow f^{\prime}$ induces a diagram

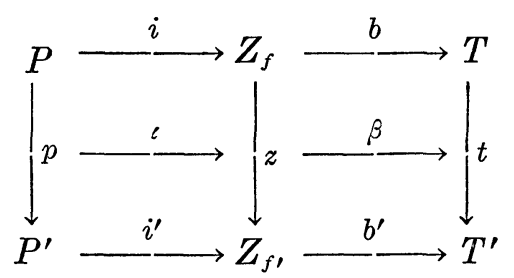

where $\iota=\left(i, i^{\prime}\right): p \rightarrow z, \beta=\left(b, b^{\prime}\right): z \rightarrow t$ and $\beta \cdot \iota=\phi$, which in turn yields the push-out diagram

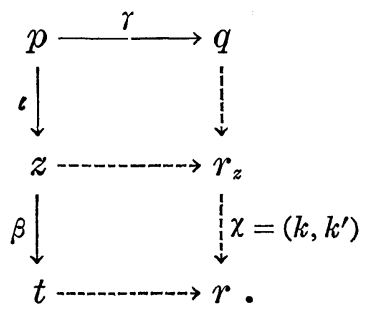

We have

LEMMA 1.7. If $t$ is a fibration, if $f^{\prime}$ is a cofibration, if $p$ is the pull-back of $t$ over $f^{\prime}$ and if $\gamma$ is a WHEF then $\chi$ is a WHEF.

Proof of 1.7. For any map $m: M^{\circ} \rightarrow M^{\prime}$ and any $x \in M^{\prime}$, let $F(m, x)$ denote the fibre of $m$ lying above $x$. Let $y \in Z\left(f^{\prime}, g^{\prime}\right)$ and let

$$
k^{\prime \prime}: F\left(r_{z}, y\right) \longrightarrow F\left(r, k^{\prime} y\right)
$$

be the map induced by $\chi$. There are three cases. If $y=\{x\}$ where $x \in Q^{\prime}$ and $x \neq g^{\prime} x^{\prime}$ for any $x^{\prime} \in P^{\prime}$ then clearly $F\left(r_{z}, y\right)$ and $F\left(r, k^{\prime} y\right)$ are homeomorphic with $F(q, x)$. Similarly if $y=\{x\}$, where $x \in T^{\prime}$ and $x \neq f^{\prime} x^{\prime}$ for any $x^{\prime} \in P^{\prime}$ then $F\left(r_{z}, y\right)$ and $F\left(r, k^{\prime} y\right)$ are homeomorphic. Suppose that

$$
\begin{array}{r}
y=\{x, s\}\left(x \in P^{\prime}, s \in I\right) \text { and let } \\
{[x]=\left\{x^{\prime} \in P^{\prime} \mid g^{\prime} x^{\prime}=g^{\prime} x\right\} .}
\end{array}
$$

Since $f^{\prime}$, being a cofibration, is injective [16], there is a push-out diagram

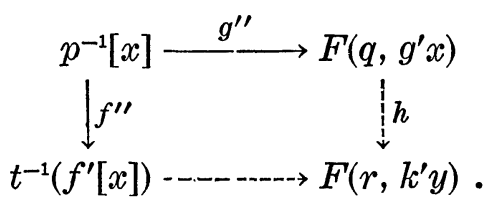


Then $f^{\prime \prime}$ is actually an equivalence: it is injective since $f$ is injective, and surjective since $P$ is a pull-back. It follows that $h$ is an equivalence. If $0 \leqq s<1$ then $F\left(r_{z}, y\right) \approx F(p, x)$ and $k^{\prime \prime}$ is equivalent to $g \mid F(p, x): F(p, x) \rightarrow F\left(q, g^{\prime} x\right)$ which is a weak homotopy equivalence. Similarly if $s=1$ then $k^{\prime \prime}$ is an equivalence. Hence $\chi$ is a WHEF.

2. Proper cofibration. A map $i: A \rightarrow X$ is a cofibration if for any map $f: X \rightarrow Y$ and any homotopy $g_{t}: A \rightarrow Y$ such that $g_{0}=f \cdot i$ there exists a homotopy $f_{t}: X \rightarrow Y$ such that $f_{0}=f$ and $g_{t}=f_{t} \cdot i$. If, further, $i(A)$ is a closed subset of $X$ then $i$ is a closed cofibration. The following lemma is [16; Th. 2].

LEMMA 2.1. $i$ is a closed cofibration if and only if there exist

(i) a neighborhood $U$ of $i(A)$ and a homotopy $H$ : $U \times I \rightarrow X$ such that $H(x, 0)=x, H(a, t)=a, H(x, 1) \in \mathrm{i}(A) \quad(x \in U, a \in \mathrm{i}(A), t \in I)$;

(ii) a map $u: X \rightarrow I$ such that $i(A)=u^{-1}(0)$ and $u x=1$ for all $x \in X-U$.

If $U, H$ and $u$ exist and satisfy the further conditions $U=u^{-1}([0,1))$, $H(U \times I) \subseteq U$ then $i$ is a proper cofibration. If $f: P \rightarrow T$ is any map, we remark that the associated map $i: P \rightarrow Z_{f}$ is a proper cofibration, for we may set $u\{x, t\}=t, u\{y\}=1, H(\{x, t\}, s)=\{x, t s\}(x \in P$, $y \in T, s, t \in I)$.

Let $f, g, \bar{f}, \bar{g}$ be as in diagram 1.1. We have

Lemma 2.2. If $f$ is a proper cofibration then so is $\bar{f}$.

Proof. Let $H$ and $u$ represent $f$ as a proper cofibration and let $\bar{u}: R \rightarrow I$ be such that

$$
\bar{u}\{x\}=u x(x \in T), \bar{u}\{y\}=0(y \in Q) .
$$

Then $\bar{u}$ is well-defined and continuous and if we let $\bar{U}=\bar{u}^{-1}([0,1))$ and let $\bar{H}: \bar{U} \times I \rightarrow R$ be such that

$$
\bar{H}(\{x\}, s)=\{H(x, s)\}, \bar{H}(\{y\}, s)=\{y\}(x \in U, y \in Q, s \in I),
$$

then $\bar{H}$ and $\bar{u}$ represent $\bar{f}$ as a proper cofibration. We remark further that there is a commutative diagram:




Now let $\phi=\left(f, f^{\prime}\right)=p \rightarrow t$ be a pair map. $\phi$ is a proper cofibration if $H$ and $u$ (resp. $H^{\prime}$ and $u^{\prime}$ ) represent $f$ (resp. $f^{\prime}$ ) as a proper cofibration and are such that the following diagram is commutative.

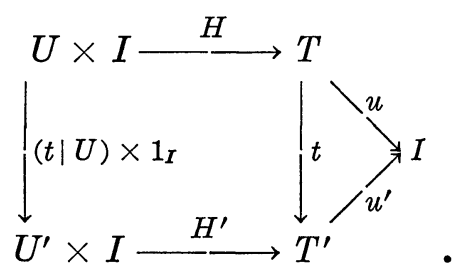

Thus the map $(f, \bar{f}): g \rightarrow \bar{g}$ in the situation of lemma 2.2 yields an example of proper cofibration. It is easy to check that another example is the pair map $\iota: p \rightarrow z$ in diagram 1.6. If $\phi$ is a proper cofibration note that, since $f(P)=u^{-1}(0)$ and $f^{\prime}\left(P^{\prime}\right)=u^{\prime-1}(0)$, it is necessarily the case that $f(P)$ is saturated with respect to $t$. One may also prove that $z$ is equivalent to a retract of $t \times 1_{I}$, from which it follows that $\phi$ is a cofibre map in the sense of Eckmann-Hilton $[4 ; p 74]$. As we shall not rely on these facts the details are omitted. We shall however require the following pair analogue of 2.2 concerning the push-out diagram

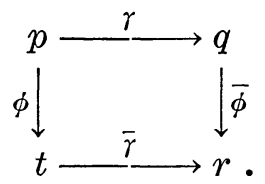

LEMMA 2.5. If $\phi$ is a proper cofibration then so is $\bar{\phi}$.

Proof. Let $H, H^{\prime}, u, u^{\prime}$ represent $\phi$ as a proper cofibration, let $\bar{H}$ and $\bar{u}$ be defined as in the proof 2.2 and let $\bar{H}^{\prime}$ and $\bar{u}^{\prime}$ be given by analogous formulae. Then in view of 2.2 it is only necessary to check the commutativity of

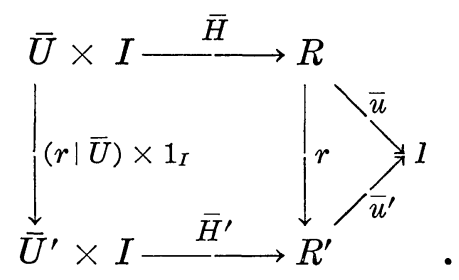

However this follows easily from the commutativity of diagram 2.4 and the relevant definitions.

Our chief reason for introducing the concept of proper cofibration is the following lemma.

LeMma 2.7. If $t$ is a fibration, if $q$ is a quasifibration, if $\phi$ : 
$p \rightarrow t$ is a proper cofibration, if $\phi$ is a WHEF and if $\gamma$ is a WHEF then $r$ is a quasifibration.

Proof. Since $\bar{\phi}$ is a proper cofibration, $\bar{f}$ and $\bar{f}^{\prime}$ are cofibrations and hence injective maps. Moreover $\bar{f}(Q)$ is saturated with respect to $r$ and since $q$ is a quasifibration it follows that $\bar{f}^{\prime}\left(Q^{\prime}\right)=\bar{u}^{\prime-1}(0)=B^{\prime}$ (say) is distinguished in the sense of Dold-Thom [3]. We claim that $\bar{U}^{\prime}$ is also distinguished, for $\bar{H}$ and $\bar{H}^{\prime}$ define homotopies $D_{s}: \bar{U} \rightarrow \bar{U}$, $d_{s}: \bar{U}^{\prime} \rightarrow \bar{U}^{\prime}$ with $D_{0}=1, D_{1}(\bar{U}) \subseteq \bar{f}(Q)=E^{\prime}$ (say), $D_{s}\left(E^{\prime}\right) \subseteq E^{\prime}, d_{0}=$ $1, d_{1}\left(\bar{U}^{\prime}\right) \subseteq B^{\prime}, d_{s}\left(B^{\prime}\right) \subseteq B^{\prime}$. In view of $[3 ; 2.10]$ it will be sufficient to prove, for each $x \in \bar{U}^{\prime}$ and $i \geqq 0$, that

$$
D_{1_{k}}: \pi_{i}(F(r, x)) \approx \pi_{i}\left(F\left(r, d_{1} x\right)\right) .
$$

2.8 is certainly satisfied if $x \in B^{\prime}$. Suppose that $x \in \bar{U}^{\prime}-\bar{f}^{\prime}\left(Q^{\prime}\right)$. Then $x=\{y\}$ where $y \in U^{\prime}-f^{\prime}\left(P^{\prime}\right)$ and $\bar{\gamma}$ induces a homeomorphism $\bar{g}^{\prime \prime}$ : $F(t, y) \rightarrow F(r, x)$. Let $k_{s}: U \rightarrow U$ and $k_{s}^{\prime}: U^{\prime} \rightarrow U^{\prime}$ be the homotopies associated with $H$ and $H^{\prime}$ respectively. Then commutativity in 2.4 yields $t \cdot k_{s}=k_{s}^{\prime} \cdot t$ so that if $y^{\prime}=k_{1}^{\prime} y$ we have a map

$$
\left(k_{1} \mid F(t, y)\right): F(t, y) \longrightarrow F\left(t, y^{\prime}\right) \text {. }
$$

Commutativity in $2.3^{\prime}$ yields $\bar{g}^{\prime} \cdot k_{s}^{\prime}=d_{s} \cdot \bar{g}^{\prime}$, hence $\bar{g}^{\prime} y^{\prime}=d_{1} x$ and we have an induced map

$$
\left(\bar{g} \mid F\left(t, y^{\prime}\right)\right): F\left(t, y^{\prime}\right) \longrightarrow F\left(r, d_{1} x\right) .
$$

Further, commutativity in 2.3 yields $\bar{g} \cdot k_{s}=D_{s} \cdot \bar{g}$ so that we have commutativity in

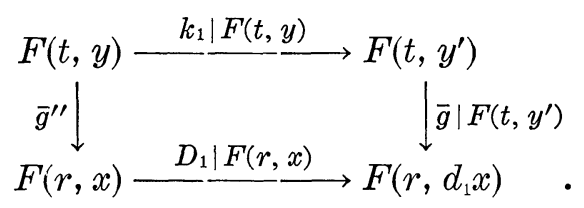

But $t$ is a fibration, hence $k_{1 . *}: \pi_{i}\left(F(t, y) \approx \pi_{i}\left(F\left(t, y^{\prime}\right)\right)\right.$, by [12; 2.8.13]. Since $\bar{g}^{\prime \prime}$ is a homeomorphism it will be sufficient to prove $\bar{g}_{*}$ : $\pi_{i}\left(F\left(t, y^{\prime}\right)\right) \approx \pi_{i}\left(F\left(r, d_{1} x\right)\right)$. Since $d_{1} x=\bar{g}^{\prime} y^{\prime} \in B^{\prime}$, there exists $x^{\prime} \in P^{\prime}$ such that $f^{\prime} x^{\prime}=y^{\prime}$ and hence we have an induced diagram

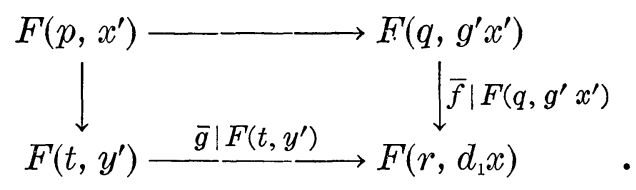

Since $\bar{f}$ is injective and $\bar{f}(Q)$ is saturated with respect to $r, \bar{f} \mid F\left(q, g^{\prime} x^{\prime}\right)$ is a homeomorphism and the remaining arrows induce homotopy isomorphisms since $\gamma$ and $\phi$ are both WHEF. To complete the proof 
of 2.7 we may observe that $R^{\prime}-B^{\prime}$ is open, for $\bar{f}^{\prime}$ is a closed cofibration. Moreover $R^{\prime}-B^{\prime}$ is distinguished since $\left(\bar{g}, \bar{g}^{\prime}\right)$ induces an equivalence of a restriction of $t$ with $r \mid r^{-1}\left(R^{\prime}-B^{\prime}\right)$. Similarly $\bar{U}^{\prime} \cap\left(R^{\prime}-B^{\prime}\right)$ is distinguished and hence 2.7 follows from [3; Satz 2.2].

We may also complete the proof of 0.2 , for it follows from 2.7 that $r_{z}$ is a quasifibration. By $1.2, k$ and $k^{\prime}$ are homotopy equivalences and, by 1.7, $\chi$ is a WHEF. A standard argument using the 5-lemma now shows that $r$ is a quasifibration.

Michael McCord has shown [7] that many of the proofs of [3] can be modified so as to apply to weak homotopy equivalences. Let $p: E \rightarrow B$ be a map and let $U$ be a subset of $B$. Then $U$ is distinguished in the sense of McCord if $p \mid p^{-1}(U): p^{-1}(U) \rightarrow U$ is a weak homotopy equivalence. We shall need the following simple analogue of $[3 ; 2.10]$.

Lemma 2.9. Let $p: E \rightarrow B$ be a continuous map onto $B$. Let $B^{\prime}$ be distinguished and let $E^{\prime}=p^{-1}\left(B^{\prime}\right)$. If there exist homotopies $D_{t}: E \rightarrow E, d_{t}: B \rightarrow B$ with $D_{0}=1_{E}, D_{t}\left(E^{\prime}\right) \subseteq E^{\prime}, D_{1}(E) \subseteq E^{\prime}, d_{0}=1_{B}$, $d_{t}\left(B^{\prime}\right) \subseteq B^{\prime}, d_{1}(B) \subseteq B^{\prime}$ and with $p \cdot D_{1}=d_{1} \cdot p$, then $B$ itself is distinguished.

Proof. For any $x \in B$ and any $y \in p^{-1}(x)$ we have a commutative diagram

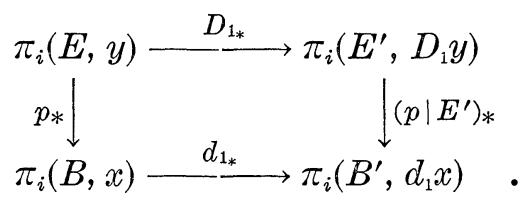

Since three of the arrows are isomorphisms, the fourth is also.

A weak homotopy equivalence analogue of 2.7 is as follows.

Lemma 2.10. If $f: P \rightarrow T$ is a proper cofibration, if $\dot{\phi}=(f, f)$ : $1_{P} \rightarrow 1_{T}$, if $q$ is a weak homotopy equivalence and if $\gamma: 1_{P} \rightarrow q$ is any map then $r$ is a weak homotopy equivalence.

Proof. $\dot{\phi}$ is certainly a proper cofibration. As in the proof of 2.7, but this time using 2.9 , we may show that $\bar{U}^{\prime}$ is distinguished. Again as in the proof of 2.7, $R^{\prime}-B^{\prime}$ and $\bar{U}^{\prime} \cap\left(R^{\prime}-B^{\prime}\right)$ are distinguished. Thus 2.10 follows from [7; Th. 6].

Proof of 0.3. 2.10 implies that $r_{z}$ is a weak homotopy equivalence. Thus 0.3 follows by two applications of 1.2 . 


\section{REFERENCES}

1. G. Allaud, On the classification of fibre spaces, Math. Zeit. 92 (1966), 110-125.

2. A. Dold and R. Lashof, Principal quasi fibrations and fibre homotopy equivalence, Illinois J. Math. 3 (1959), 285-305.

3. A. Dold and R. Thom, Quasifaserungen und unendliche symmetrische Produkte, Ann. of Math. 67 (1958), 239-281.

4. P. Hilton, Homotopy Theory and Duality, Gordon and Breach, 1965.

5. Y. Husseini, When is a complex fibred by a subcomplex?, Trans. Amer. Math. Soc. 124 (1966), 249-291.

6. I. M. James, The transgression and Hopf invariant of a fibration, Proc. London Math. Soc. (3) 11 (1961), 588-600.

7. M. C. McCord, Singular homology groups and homotopy groups of finite topological spaces, Duke Math. J. 33 (1966), 465-474.

8. R. J. Milgram, The bar construction and abelian H-spaces, Illinois J. Math. 11 (1967), 242-250.

9. J. Milnor, Construction of universal bundles II, Ann. of Math. (2) 63 (1956), 430436.

10. Y. Nomura, An application of the path-space technique to the theory of triads, Nagoya Math. J. 22 (1963), 169-188.

11. D. Puppe, Bemerkungen ïber die Erweiterung von Homotopien, Archiv. der Math. 18 (1967), 81-88.

12. E. H. Spanier, Algebraic Topology, McGraw-Hill, 1966.

13. J. Stasheff, A classification theorem for fibre spaces, Topology 2 (1963), 239-246.

14. - Associated fibre spaces, Michigan Math. J. 15 (1968), 457-470.

15. N. E. Steenrod, Milgram's classifying space for a topological group, Topology 7 (1968), 349-368.

16. A. Str $\phi \mathrm{m}$, Note on cofibrations, Math. Scand. 19 (1966), 11-14.

17. —, Note on cofibrations II, Math. Scand. 22 (1968), 130-142.

Received January 6, 1970. Prepared with the assistance of University of Cape Town Staff Research Grant 46277 and of South African Council for Scientific and Industrial Research Grant 40/332.

UNIVERSITY OF CAPE TOWN

Republic of South AFrica 



\section{PACIFIC JOURNAL OF MATHEMATICS}

\section{EDITORS}

\author{
H. SAMELSON \\ Stanford University \\ Stanford, California 94305

\section{Richard Pierce} \\ University of Washington \\ Seattle, Washington 98105
}

J. DugundJI

Department of Mathematics

University of Southern California

Los Angeles, California 90007

RichaRd ARENS

University of California

Los Angeles, California 90024

\section{ASSOCIATE EDITORS}
E. F. BECKENBACH
B. H. NeUMANN
F. WOLE
K. YoshidA

\section{SUPPORTING INSTITUTIONS}

\author{
UNIVERSITY OF BRITISH COLUMBIA \\ CALIFORNIA INSTITUTE OF TECHNOLOGY \\ UNIVERSITY OF CALIFORNIA \\ MONTANA STATE UNIVERSITY \\ UNIVERSITY OF NEVADA \\ NEW MEXICO STATE UNIVERSITY \\ OREGON STATE UNIVERSITY \\ UNIVERSITY OF OREGON \\ OSAKA UNIVERSITY \\ UNIVERSITY OF SOUTHERN CALIFORNIA
}

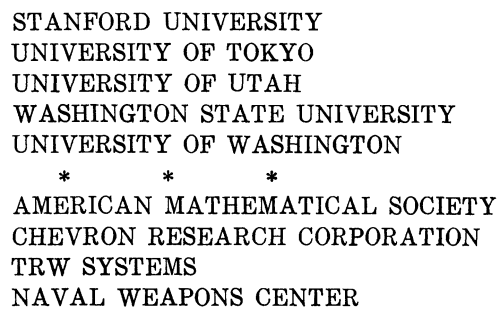

The Supporting Institutions listed above contribute to the cost of publication of this Journal, but they are not owners or publishers and have no responsibility for its content or policies.

Mathematical papers intended for publication in the Pacific Journal of Mathematics should be in typed form or offset-reproduced, (not dittoed), double spaced with large margins. Underline Greek letters in red, German in green, and script in blue. The first paragraph or two must be capable of being used separately as a synopsis of the entire paper. The editorial "we" must not be used in the synopsis, and items of the bibliography should not be cited there unless absolutely necessary, in which case they must be identified by author and Journal, rather than by item number. Manuscripts, in duplicate if possible, may be sent to any one of the four editors. Please classify according to the scheme of Math. Rev. Index to Vol. 39. All other communications to the editors should be addressed to the managing editor, Richard Arens, University of California, Los Angeles, California, 90024.

50 reprints are provided free for each article; additional copies may be obtained at cost in multiples of 50 .

The Pacific Journal of Mathematics is published monthly. Effective with Volume 16 the price per volume (3 numbers) is $\$ 8.00$; single issues, $\$ 3.00$. Special price for current issues to individual faculty members of supporting institutions and to individual members of the American Mathematical Society: $\$ 4.00$ per volume; single issues $\$ 1.50$. Back numbers are available.

Subscriptions, orders for back numbers, and changes of address should be sent to Pacific Journal of Mathematics, 103 Highland Boulevard, Berkeley, California, 94708.

PUBLISHED BY PACIFIC JOURNAL OF MATHEMATICS, A NON-PROFIT CORPORATION

Printed at Kokusai Bunken Insatsusha (International Academic Printing Co., Ltd.), 7-17, Fuj̣imi 2-chome, Chiyoda-ku, Tokyo, Japan. 


\section{Pacific Journal of Mathematics}

\section{Vol. 35, No. $2 \quad$ October, 1970}

Valentin Danilovich Belousov and Palaniappan L. Kannappan, Generalized Bol functional equation .................................... 259

Charles Morgan Biles, Gelfand and Wallman-type compactifications ........... 267

Louis Harvey Blake, A generalization of martingales and two consequent convergence theorems .................................... 279

Dennis K. Burke, On p-spaces and $w \Delta$-spaces..................... 285

John Ben Butler, Jr., Almost smooth perturbations of self-adjoint operators . . . . . . 297

Michael James Cambern, Isomorphisms of $C_{0}(Y)$ onto $C(X) \ldots \ldots \ldots \ldots \ldots . \ldots 307$

David Edwin Cook, A conditionally compact point set with noncompact closure ... 313

Timothy Edwin Cramer, Countable Boolean algebras as subalgebras and homomorphs .........................................

John R. Edwards and Stanley G. Wayment, A v-integral representation for linear operators on spaces of continuous functions with values in topological vector spaces.............................................

Mary Rodriguez Embry, Similarities involving normal operators on Hilbert

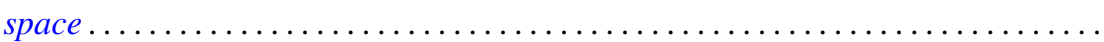

Lynn Harry Erbe, Oscillation theorems for second order linear differential

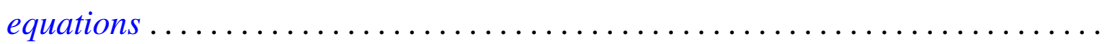

William James Firey, Local behaviour of area functions of convex bodies .......... Joe Wayne Fisher, The primary decomposition theory for modules ..............

Gerald Seymour Garfinkel, Generic splitting algebras for Pic ..................

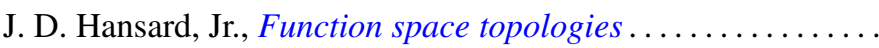

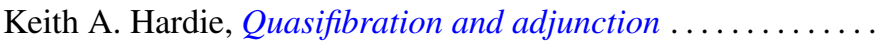

G. Hochschild, Coverings of pro-affine algebraic groups ...........

Gerald L. Itzkowitz, On nets of contractive maps in uniform spaces ..

381

389

399

417

Melven Robert Krom and Myren Laurance Krom, Groups with free nonabelian subgroups....................................

James Robert Kuttler, Upper and lower bounds for eigenvalues by finite differences ......................................

Dany Leviatan, A new approach to representation theory for convolution transforms . . .

Richard Beech Mansfield, Perfect subsets of definable sets of real numbers ...

Brenda MacGibbon, A necessary and sufficient condition for the embedding of a

Lindelof space in a Hausdorff $\mathscr{H} \sigma$ space ..................

David G. Mead and B. D. McLemore, Ritt's question on the Wronskian ....

Edward Yoshio Mikami, Focal points in a control problem .....

Paul G. Miller, Characterizing the distributions of three independent n-dimensional random variables, $X_{1}, X_{2}, X_{3}$, having analytic characteristic functions by the joint distribution of $\left(X_{1}+X_{3}, X_{2}+X_{3}\right)$. . .

P. Rosenthal, On the Bergman integral operator for an elliptic partial differential equation with a singular coefficient....

Douglas B. Smith, On the number of finitely generated $O$-group 